\title{
Upper Unidomination Number and Upper Total Unidomination Number of a 3-Regularized Wheel
}

\author{
V. Anantha Lakshmi \\ Lecturer, Department of Mathematics, \\ P.R.Govt. College (A), Kakinada-533004, \\ Andhra Pradesh, India
}

\author{
B. Maheswari \\ Professor, Department of Applied Mathematics, \\ S.P.Mahila Visvavidyalayam, Tirupati-517502, \\ Andhra Pradesh, India
}

\begin{abstract}
The concept of unidominating function and total unidominating functions are introduced in [5] and [6] respectively.Minimal unidominating function and upper unidominationnumber are introduced in [7] and minimal total unidominating function and upper total unidomination number are introduced in [8]. The unidomination number and total unidomination number of a 3-regularized wheel are obtained in [9], [10]. In this paper the authors study the minimal unidominating functions, minimal total unidominating functions of a 3-regularized wheel and determined its upper unidomination number, upper total unidomination number. Further the number of minimal unidominating functions, minimal total unidominating functions with maximum weight are found.
\end{abstract}

\section{Keywords}

Wheel, 3-regularized wheel, minimal unidominating function, minimal total unidominating function, upper unidomination number, upper total unidomination number.

\section{INTRODUCTION}

Graph Theory plays an important role in several areas of computer science such as artificial intelligence, formal languages, computer graphics etc. An important branch of graph theory is domination and its properties have been widely studied by T.W.Haynes and others in [1,2]. Hedetniemi [3] introduced the concept of dominating function which has many applications. Zelinka, B [4] has given some remarks on domination in cubic graphs. In [5], [6] the concepts of unidominating function and total unidominating function are introduced by the authors. The concept of minimal unidominating function and upper unidominationnumber are introduced by the authors in [7] and minimal total unidominating function and upper total unidomination are introduced in [8]. In [9], [10] the unidomination number and total unidomination number of a 3-regularized wheel are obtained. In this paper the upper unidomination number and upper total unidomination number of a 3-Regularized Wheel are found. Further the number of minimal unidominating functions and minimal total unidominating functions with maximum weight for this graph are obtained and the results obtained are illustrated.

\section{DEFINITIONS}

In this section the concepts of unidominating function, minimal unidominating function, upper unidomination number, total unidominating function, minimal total unidominating function, and upper total unidomination number are defined as follows.

Definition 2.1: Let $G(V, E)$ be a graph. A function $f: V \rightarrow\{0,1\}$ is said to be anunidominating function

$$
\begin{aligned}
& \text { if } \sum_{u \in N[v]} f(u) \geq 1 \quad \forall v \in \operatorname{Vand} f(v)=1, \\
& \sum_{u \in N[v]} f(u)=1 \quad \forall v \in \operatorname{Vand} f(v)=0 .
\end{aligned}
$$

where $N[v]$ is the closed neighbourhood of the vertex $v$.

Definition 2.2: Let $G(V, E)$ be a graph. An unidominating function $f: V \rightarrow\{0,1\}$ is called a minimal unidominating function if for all $g<f, g$ is not an unidominating function.

Definition 2.3: The upper unidomination number of a graph $G(V, E)$ is defined as $\max \{f(V) / f$ is a minimal unidominating function $\}$,

where $f(V)=\sum_{u \in V} f(u)$.

The upper unidomination number of $\mathrm{G}$ is denoted by $\Gamma_{u}(G)$.

Definition 2.4: Let $G(V, E)$ be a connected graph. A function $f: V \rightarrow\{0,1\}$ is said to be a total unidominating function, if

$$
\begin{gathered}
\sum_{u \in N(v)} f(u) \geq 1 \forall v \in V \text { and } f(v)=1, \\
\sum_{u \in N(v)} f(u)=1 \forall v \in V \text { and } f(v)=0,
\end{gathered}
$$

where $N(v)$ is the open neighbourhood of the vertex $v$.

Definition 2.5: Let $G(V, E)$ be a connected graph. A total unidominating function $f: V \rightarrow\{0,1\}$ is called a minimal total unidominating function if for all $g<f, g$ is not a total unidominating function.

Definition 2.6: The upper total unidomination number of a connected graph $G(V, E)$ is defined as $\max \{f(V) / f$ is a minimal total unidominating function $\}$.

It is denoted by $\Gamma_{t u}(G)$.

Definition 2.7: A 3- Regularized wheel is defined as "A graph formed from $W_{1, n}$ by replacing the center of $W_{1, n}$ by a cycle $C_{n}$ and each of the remaining $n$ vertices in $W_{1, n}$ are replaced by cycles $C_{3}$ ".

\section{UPPER UNIDOMINATION NUMBER OF A 3-REGULARIZED WHEEL}

In this section the upper unidomination number of a 3regularised wheel and the number of minimal unidominating functions with maximum weight are obtained. 
Theorem 3.1: The upper unidomination number of a 3regularized wheel formed from $W_{1, n}$ is

$$
\left\{\begin{array}{l}
\frac{5 n}{2} \quad \text { if } n \text { is even, } \\
\left\lfloor\frac{5 n}{2}\right\rfloor-1 \quad \text { if } n \text { is odd. }
\end{array}\right.
$$

Proof: Let $W_{1, n}$ be a wheel and $C_{n}$ be the cycle replacing the center of $W_{1, n}$ and $C_{3}^{1}, C_{3}^{2}, \ldots, C_{3}^{n}$ are the cycles replacing the $n$ vertices in $W_{1, n}$ respectively.

Let $u_{1}, u_{2}, \ldots \ldots, u_{n}$ be the vertices in $C_{n}$, and $v_{1}, v_{2}, \ldots ., v_{n}$ be the vertices in $C_{3}^{1}, C_{3}^{2}, \ldots, C_{3}^{n}$ respectively which are adjacent to $u_{1}, u_{2}, \ldots \ldots, u_{n}$ respectively. Let $w_{1}, w_{2} ; w_{3}, w_{4} ; \ldots ; w_{2 n-1}, w_{2 n}$ be the remaining vertices in $C_{3}^{1}, C_{3}^{2}, \ldots, C_{3}^{n}$ respectively.

Here $d\left(u_{i}\right)=d\left(v_{i}\right)=d\left(w_{2 i}\right)=d\left(w_{2 i-1}\right)=3$

for $i=1,2, \ldots, n$.

The upper unidomination number of this 3-regularized wheel is found in the following two cases.

Case 1: Let $n$ be an even number.

Define a function $f: V \rightarrow\{0,1\}$ by

$f(u)=\left\{\begin{array}{r}1 \quad \text { when } u=u_{i}, i=1,2, \ldots, n, u=v_{j}, w_{2 j-1}, w_{2 j} \\ \text { when } j \text { is an odd number, }\end{array}\right.$

Sub case 1: Let $i$ be an even number.

$$
\begin{gathered}
\text { Then } \sum_{u \in N\left[u_{i}\right]} f(u)=f\left(u_{i-1}\right)+f\left(u_{i}\right)+f\left(u_{i+1}\right)+f\left(v_{i}\right) \\
=1+1+1+0=3>1, \\
\sum_{u \in N\left[v_{i}\right]} f(u)=f\left(u_{i}\right)+f\left(v_{i}\right)+f\left(w_{2 i-1}\right)+f\left(w_{2 i}\right) \\
=1+0+0+0=1, \\
\sum_{u \in N\left[w_{2 i-1}\right]} f(u)=f\left(w_{2 i-1}\right)+f\left(v_{i}\right)+f\left(w_{2 i-2}\right)+f\left(w_{2 i}\right) \\
=0+0+1+0=1, \\
\sum_{u \in N\left[w_{2 i}\right]} f(u)=f\left(w_{2 i}\right)+f\left(v_{i}\right)+f\left(w_{2 i-1}\right)+f\left(w_{2 i+1}\right) \\
=0+0+0+1=1 .
\end{gathered}
$$

Sub case 2: Let $i$ be an odd number.

$$
\begin{gathered}
\text { Then } \sum_{u \in N\left[u_{i}\right]} f(u)=f\left(u_{i-1}\right)+f\left(u_{i}\right)+f\left(u_{i+1}\right)+f\left(v_{i}\right) \\
=1+1+1+1=4>1, \\
\sum_{u \in N\left[v_{i}\right]} f(u)=f\left(u_{i}\right)+f\left(v_{i}\right)+f\left(w_{2 i-1}\right)+f\left(w_{2 i}\right) \\
=1+1+1+1=4>1, \\
\sum_{u \in N\left[w_{2 i-1}\right]} f(u)=f\left(w_{2 i-1}\right)+f\left(v_{i}\right)+f\left(w_{2 i-2}\right)+f\left(w_{2 i}\right) \\
=1+1+0+1=3>1, \\
\sum_{u \in N\left[w_{2 i}\right]} f(u)=f\left(w_{2 i}\right)+f\left(v_{i}\right)+f\left(w_{2 i-1}\right)+f\left(w_{2 i+1}\right) \\
=1+1+1+0=3>1,
\end{gathered}
$$

Therefore $f$ is a unidominating function.

$$
\text { Now } \begin{aligned}
f(V)=\sum_{u \in V} f(u)=\sum_{i=1}^{n}\left[f\left(u_{i}\right)+f\left(v_{i}\right)\right] \\
\quad+\sum_{i=1}^{2 n} f\left(w_{i}\right)=n+\frac{n}{2}+n=\frac{5 n}{2} .
\end{aligned}
$$

Now we check for the minimality of $f$.

Now we define a function $g$ such that $g<f$, and show that $g$ is not a unidominating function for all possibilities of defining $g$.

Case (i) : Define a function $g: V \rightarrow\{0,1\}$ by

$g(u)=f(u)$ for all $u \in V, u \neq u_{k}, u_{k+1}$

for some $k \in\{1,2, \ldots, n\}$

and $g\left(u_{k}\right)=g\left(u_{k+1}\right)=0$.

Then obviously $g<f$.

Sub case 1: Let $k$ be even.

Then $g\left(v_{k}\right)=g\left(w_{2 k-1}\right)=g\left(w_{2 k}\right)=0$.

But $\sum_{u \in N\left[v_{k}\right]} g(u)=g\left(u_{k}\right)+g\left(v_{k}\right)+g\left(w_{2 k-1}\right)+g\left(w_{2 k}\right)$

$$
=0+0+0+0=0 \neq 1 .
$$

Sub case 2: Let $k$ be odd. Then $k+1$ is even and hence

$$
g\left(v_{k+1}\right)=g\left(w_{2 k+1}\right)=g\left(w_{2 k+2}\right)=0 .
$$

$$
\text { But } \begin{aligned}
\sum_{u \in N\left[v_{k+1}\right]} g(u) & =g\left(u_{k+1}\right)+g\left(v_{k+1}\right)+g\left(w_{2 k+1}\right) \\
& +g\left(w_{2 k+2}\right)=0 \neq 1 .
\end{aligned}
$$

Therefore it follows that $g$ is not a unidominating function.

Case (ii): Define another function $g_{1}: V \rightarrow\{0,1\}$ by

$g_{1}(u)=f(u)$ for all $u \in V, u \neq w_{2 k-1}, w_{2 k}$ for some $k \in\{1,2, \ldots, n\}$, $k$ is odd,

and so $g_{1}\left(w_{2 k-1}\right)=g_{1}\left(w_{2 k}\right)=0$.

Since $k$ is odd, $k-1$ is even so that

$g_{1}\left(v_{k-1}\right)=g_{1}\left(w_{2 k-3}\right)=g_{1}\left(w_{2 k-2}\right)=0$.

Now $\sum_{u \in N\left[w_{2 k-2}\right]} g_{1}(u)$

$$
\begin{aligned}
& =g_{1}\left(w_{2 k-3}\right)+g_{1}\left(w_{2 k-2}\right)+g_{1}\left(w_{2 k-1}\right) \\
& +g_{1}\left(v_{k-1}\right)=0 \neq 1
\end{aligned}
$$

Therefore $g_{1}$ is not a unidominating function.

Case (iii): Define another function $g_{2}: V \rightarrow\{0,1\}$ by $g_{2}(u)=f(u)$ for all $u \in V, u \neq v_{k}$

for some $k \in\{1,2, \ldots, n\}, k$ is odd,

And so $g_{2}\left(v_{k}\right)=0$.

Now $\begin{aligned} \sum_{u \in N\left[v_{k}\right]} g_{2}(u) & =g_{2}\left(u_{k}\right)+g_{2}\left(v_{k}\right)+g_{2}\left(w_{2 k-1}\right) \\ & +g_{2}\left(w_{2 k}\right)=1+0+1+1=3 \neq 1\end{aligned}$

Therefore $g_{2}$ is not a unidominating function.

Case (iv): Define another function $g_{3}: V \rightarrow\{0,1\}$ by

$g_{3}(u)=f(u)$ for all $u \in V, u \neq v_{k}, w_{2 k-1}, w_{2 k}$ 
for some $k \in\{1,2, \ldots, n\}, \quad k$ is odd,

and so $g_{3}\left(v_{k}\right)=0, g_{3}\left(w_{2 k-1}\right)=g_{3}\left(w_{2 k}\right)=0$.

Then by the same argument for $g_{1}$ in Case (ii), it follows that $g_{3}$ is not a unidominating function.

Since all these functions are defined arbitrarily and as there is no other possibility of defining a function $<f$, it follows that $f$ is a minimal unidominating function

Thus $\Gamma_{u}(3-$ regularizedwheel $) \geq \frac{5 n}{2}----(1)$

Consider a minimal unidominating function $f$ of a 3regularised wheel. Then it must satisfy the following conditions, otherwise it cannot be a minimal unidominating function.

1. If $f\left(u_{i}\right)=1$ for all $i \in\{1,2, \ldots, n\}$ then all $v_{i}^{\prime} s$ should not be assigned 1 and no two consecutive $v_{i}$ 's can have functional value 1 .

2.

If $f\left(u_{i}\right)=1$ and $f\left(v_{i}\right)=0$ then

$f\left(w_{2 i-1}\right)=f\left(w_{2 i}\right)=0$.

3. If $f\left(v_{i}\right)=1$ then either

$f\left(w_{2 i-1}\right)=f\left(w_{2 i}\right)=1 \operatorname{orf}\left(w_{2 i-1}\right)=f\left(w_{2 i}\right)=0$.

4. If $f\left(u_{i}\right)=0$ and any one of $f\left(u_{i-1}\right)$ or $f\left(u_{i+1}\right)$ is 1 then $f\left(v_{i}\right)$ must be 0 and any one of $f\left(w_{2 i-1}\right), f\left(w_{2 i}\right)$ must be 1 .

Otherwise if $f\left(u_{i-1}\right)=f\left(u_{i}\right)=f\left(u_{i+1}\right)=0$ then $f\left(v_{i}\right)$ must be 1 and $f\left(w_{2 i-1}\right), f\left(w_{2 i}\right)$ are 0 .

Let $f$ be a minimal unidominating function of a 3 -regularized wheel.

Let $\mathrm{k}$ be the number of $u_{i} s$ which have functional value 0 . Then for each such $i$,

$$
f\left(v_{i}\right)+f\left(w_{2 i-1}\right)+f\left(w_{2 i}\right)=1 .
$$

Therefore $\sum\left[f\left(v_{i}\right)+f\left(w_{2 i-1}\right)+f\left(w_{2 i}\right)\right]$

$$
=k \text { for these } k \text { sets of vertices }
$$

$\left(v_{i}, w_{2 i-1}, w_{2 i}\right)$, where $i$ is such that $f\left(u_{i}\right)=0$.

Suppose that the number of consecutive $u_{i} s$ which have functional value 1 are $k_{1}, k_{2}, \ldots, k_{p}$ respectively.

Then $k_{1}+k_{2}+\ldots+k_{p}=n-k$,

$1 \leq k_{1}, k_{2}, \ldots, k_{p} \leq n-k, p \leq \frac{k}{2}<k$.

Then we have

$\sum\left[f\left(v_{i}\right)+f\left(w_{2 i-1}\right)+f\left(w_{2 i}\right)\right] \leq \frac{k_{1}}{2}+\frac{k_{1}}{2}+\frac{k_{1}}{2}=\frac{3 k_{1}}{2}$

if $k_{1}$ is even,

$$
=\frac{k_{1}+1}{2}+\frac{k_{1}+1}{2}+\frac{k_{1}+1}{2} \leq \frac{3\left(k_{1}+1\right)}{2} \text { if } k_{1} \text { is odd. }
$$

Similar is the case for $k_{2}, k_{3}, \ldots, k_{p}$.

Without loss of generality assume that $k_{1}, k_{2}, \ldots, k_{r}$ are even and $k_{r+1}, k_{r+2}, \ldots, k_{p}$ are odd. Then

$$
\begin{aligned}
f(V)=\sum_{i=1}^{n} f\left(u_{i}\right)+ & \sum_{k_{1}}\left[f\left(v_{i}\right)+f\left(w_{2 i-1}\right)+f\left(w_{2 i}\right)\right]+\cdots \\
& +\sum_{k_{p}}\left[f\left(v_{i}\right)+f\left(w_{2 i-1}\right)+f\left(w_{2 i}\right)\right] \\
& +\sum_{k}\left[f\left(v_{i}\right)+f\left(w_{2 i-1}\right)+f\left(w_{2 i}\right)\right] \\
\leq & n-k+\frac{3\left(k_{1}+k_{2}+\cdots+k_{r}\right)}{2} \\
+ & \frac{3\left(k_{r+1}+1+k_{r+2}+1+\ldots+k_{p}+1\right)}{2}+k \\
= & n+\frac{3(n-k+p-r)}{2}=\frac{5 n}{2}-\frac{3(k+r-p)}{2} \leq \frac{5 n}{2}
\end{aligned}
$$

Therefore for any minimal unidominating function $f$ of a 3 -regularized wheel $f(V) \leq \frac{5 n}{2}$.

Thus $\max \{f(V) / f$ is a minimal unidominating function $\} \leq \frac{5 n}{2}$.

That is $\Gamma_{\mathrm{u}}(3-$ regularized wheel $) \leq \frac{5 \mathrm{n}}{2}---$

Therefore from the inequalities (1) and (2) we have

$$
\Gamma_{\mathrm{u}}(3-\text { regularized wheel })=\frac{5 \mathrm{n}}{2}, \text { when } \mathrm{n} \text { is even. }
$$

Case 2: Let $n$ be an odd number.

Define a function $f: V \rightarrow\{0,1\}$ by

$f(u)$

$$
=\left\{\begin{array}{rr}
1 & \text { foru }=u_{i}, i=1,2, \ldots, n-2, \\
1 & \text { foru }=v_{j}, w_{2 j-1} \text { whenjisanoddnumber }, j \neq n, \\
1 & \text { for } u=w_{2 n-3}, \\
1 & \text { for } u=w_{2 j} \text { when } j \text { is an odd number }, \\
0 & \text { otherwise. }
\end{array}\right.
$$

This function is similar to the function $f$ defined in Case 1 except for the vertices $u_{n-1}, u_{n}, v_{n}, w_{2 n-3}, w_{2 n-1}$.

We can check the condition of unidominating function in the closed neighbourhood of the above vertices and see that $f$ is a unidominating function.

Now we check for the minimality of $f$.

Define a function $g: V \rightarrow\{0,1\}$ by

$g(u)=f(u)$ for all $u \in V, u \neq w_{2 n-3}$.

Then obviously $g<f$ and

$$
\begin{aligned}
\sum_{u \in N\left[w_{2 n-2}\right]} g(u)= & g\left(w_{2 n-3}\right)+g\left(w_{2 n-2}\right)+g\left(w_{2 n-1}\right) \\
& +g\left(v_{n-1}\right)=0+0+0+0=0 \neq 1
\end{aligned}
$$

Therefore $g$ is not a unidominating function.

For all possibilities of defining a function $g<f$,we can see that $g$ is not a unidominating function.

Therefore $f$ is a minimal unidominating function.

Now $f(V)=\sum_{u \in V} f(u)$

$=\sum_{i=1}^{n}\left[f\left(u_{i}\right)+f\left(v_{i}\right)+f\left(w_{2 i-1}\right)+f\left(w_{2 i}\right)\right]$ 
$=(n-2)+\frac{n-1}{2}+\frac{n-1}{2}+1+\frac{n-1}{2}+1$

$=\frac{5 n-3}{2}=\frac{5 n-1}{2}-1=\left\lfloor\frac{5 n}{2}\right\rfloor-1$.

Hence it follows that

$$
\Gamma_{\mathrm{u}}(3-\text { regularized wheel }) \geq\left\lfloor\frac{5 n}{2}\right\rfloor-1---(1)
$$

If $f$ is a minimal unidominating function then in Case 1 we have proved in general that $f(V) \leq n+\frac{3(n-k+p-r)}{2}$. That is we have not taken in to consideration that $n$ is even.

$$
\begin{aligned}
\text { Therefore } f(V) \leq & n+\frac{3(n-k+p-r)}{2} \\
& =n+\frac{3((n-1)-(k+r-p-1))}{2} \\
= & \frac{5 n-3}{2}-\frac{3(k+r-p-1)}{2} \leq \frac{5 n-3}{2}=\left\lfloor\frac{5 n}{2}\right\rfloor-1 .
\end{aligned}
$$

Thus for any minimal unidominating function $f$,

$f(V) \leq\left\lfloor\frac{5 n}{2}\right\rfloor-1$.

Hence it follows that

$$
\Gamma_{u}(3-\text { regularized wheel }) \leq\left\lfloor\frac{5 n}{2}\right\rfloor-1---(2)
$$

Therefore from the inequalities (1) and (2) we have

$$
\Gamma_{u}(3-\text { regularized wheel })=\left\lfloor\frac{5 n}{2}\right\rfloor-1 .
$$

Theorem 3.2: The number of minimal unidominating functions with maximum weight of a 3 -regularized wheel is

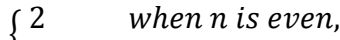

(n when $n$ is odd.

Proof: Consider a 3 -regularized wheel formed from a wheel $W_{1, n}$.

Case 1: Let $n$ be an even number.

Consider the minimal unidominating function $f$ with maximum weight $\frac{5 n}{2}$ given in Case 1 of Theorem 3.1. Then the functional values of $f$ are

$$
\begin{gathered}
f\left(u_{i}\right)=1 \forall i \in\{1,2, \ldots, n\} \\
f\left(v_{i}\right)=f\left(w_{2 i-1}\right)=f\left(w_{2 i}\right)=1 \text { for } i \text { is odd, } \\
f\left(v_{i}\right)=f\left(w_{2 i-1}\right)=f\left(w_{2 i}\right)=0 \text { for } i \text { is even. }
\end{gathered}
$$

Represent

$f\left(u_{i}\right), f\left(v_{i}\right), f\left(w_{2 i-1}\right), f\left(w_{2 i}\right)$ as $\left\{\begin{array}{l}1 \\ 0\end{array}\right.$

when $f\left(v_{i}\right)=f\left(w_{2 i-1}\right)=f\left(w_{2 i}\right)=1$ and $f\left(u_{i}\right)=1$.

when $f\left(v_{i}\right)=f\left(w_{2 i-1}\right)=f$ and $f\left(u_{i}\right)=1$.

Therefore the values of $\left(u_{i}, v_{i}, w_{2 i-1}, w_{2 i}\right), i=1,2, \ldots, n$ are represented by

$1010---10$ circularly.

Take $a-10$. Then the functional values of $f$ are in the pattern of $a a \ldots a$ circularly. These can be arranged in only one way. By the rotation of these values after the first rotation we obtain the same function $f$.
Therefore there are two minimal unidominating functions with maximum weight $\frac{5 n}{2}$.

Now we test whether there are any other minimal unidominating functions of weight $\frac{5 n}{2}$.

Let $f$ be any minimal unidominating function of a 3 -regularized wheel. Then we have proved in Case 1 of Theorem 3.1 that

$$
f(V) \leq n+\frac{3(n-k+p-r)}{2} .
$$

If $k=0$ we get $r=1, p=1$ so that $f(V)=\frac{5 n}{2}$.

That is this function coincides with one of the above said two minimal unidominating functions.

If $k=1$ then $f$ cannot be a unidominating function.

Suppose $k \geq 2$. Then $r \geq 0, p \geq 1$, and so

$$
\begin{aligned}
& k+r-p>0 \text {. Then } \\
& f(V) \leq \frac{5 n}{2}-\frac{3(k+r-p)}{2}<\frac{5 n}{2} .
\end{aligned}
$$

Then $f$ has no maximum weight.

Therefore there is no other minimal unidominating function with maximum weight $\frac{5 n}{2}$.

Case 2: Let $n$ be an odd number.

Let $f$ be a minimal unidominating function defined in Case 2 of Theorem 3.1. As in Case 1 by rotating the functional values of the vertices taking $u_{i}, v_{i}, w_{2 i-1}, w_{2 i}$ as one unit we get $n$ minimal unidominating functions.

We now verify that if there is any other minimal unidominating function with maximum weight $\frac{5 n}{2}$.

Let $f$ be a minimal unidominating function of a 3-regularized wheel. Then we have proved in Case 2 of Theorem 3.1 that

$f(V) \leq \frac{5 n-3}{2}-\frac{3(k+r-p-1)}{2}$.

If $k=0$ and 1 then $f$ can not be a minimal unidominating function. Therefore $k$ must be greater than or equal to 2 .

If $k=2$ then $p=1, r=0$. Now $f(V)=\frac{5 n-3}{2}=\left\lfloor\frac{5 n}{2}\right\rfloor-1$.

If $k>2$ then $k+r-p-1>0$, so that

$$
f(V) \leq \frac{5 n-3}{2}-\frac{3(k+r-p-1)}{2}<\frac{5 n-3}{2}=\left\lfloor\frac{5 n}{2}\right\rfloor-1 .
$$

Therefore there is no other minimal unidominating function with maximum weight

\section{UPPER TOTAL UNIDOMINATION NUMBER OF A 3-REGULARIZED WHEEL}

In this section the upper total unidomination number of a 3regularized wheel and the number of minimal total unidominating functions with maximum weight are obtained.

Theorem 4.1: The upper total unidomination number of a 3regularized wheel formed from $W_{1, n}$ is

$\Gamma_{t u}(3-$ regularized wheel $)=3 n$. 
Proof: Let $W_{1, n}$ be a wheel. Consider the 3-regularized wheel formed from $W_{1, n}$ which is defined in 2.7.

Define a function $f: V \rightarrow\{0,1\}$ by

$$
f(u)=\left\{\begin{array}{rr}
1 & \text { for } u=v_{i}, w_{2 i-1}, w_{2 i}, i=1,2, \ldots, n, \\
0 & \text { for } u=u_{i} i=1,2, \ldots, n .
\end{array}\right.
$$

Now for $i=1,2, \ldots, n$ we have

$$
\begin{gathered}
\sum_{u \in N\left(u_{i}\right)} f(u)=f\left(u_{i-1}\right)+f\left(u_{i+1}\right)+f\left(v_{i}\right)=0+0+1=1 . \\
\sum_{u \in N\left(v_{i}\right)} f(u)=f\left(u_{i}\right)+f\left(w_{2 i-1}\right)+f\left(w_{2 i}\right)=0+1+1=2 . \\
\sum_{u \in N\left(w_{2 i-1}\right)} f(u)=f\left(v_{i}\right)+f\left(w_{2 i-2}\right)+f\left(w_{2 i}\right)=1+1+1 \\
\sum_{u \in N\left(w_{2 i}\right)} f(u)=f\left(v_{i}\right)+f\left(w_{2 i-1}\right)+f\left(w_{2 i+1}\right)=1+1+1 \\
=3 .
\end{gathered}
$$

Therefore $f$ is a total unidominating function.

Now we check for the minimality of $f$.

Now we define a function $g$ such that $g<f$, and show that $g$ is not a total unidominating function for all possibilities of defining $g$.

Define a function $g: V \rightarrow\{0,1\}$ by

$g(u)=f(u) \forall u \in V$, for $u \neq v_{k}$

$$
k \in\{1,2, \ldots, n\}, \text { and } g\left(v_{k}\right)=0 .
$$

Obviously $g<f$.

$$
\text { Now } \begin{aligned}
\sum_{u \in N\left(u_{k}\right)} g(u) & =g\left(u_{k-1}\right)+g\left(u_{k+1}\right)+g\left(v_{k}\right) \\
& =0+0+0=0 \neq 1 .
\end{aligned}
$$

Therefore $g$ is not a total unidominating function.

Define another function $h: V \rightarrow\{0,1\}$ by

$h(u)=f(u) \forall u \in V$, for $u \neq w_{2 k-1}$,

$k \in\{1,2, \ldots, n\}$, and $h\left(w_{2 k-1}\right)=0$.

Obviously $h<f$.

$$
\text { Now } \begin{aligned}
\sum_{u \in N\left(w_{2 k-1}\right)} h(u) & =h\left(w_{2 k}\right)+h\left(w_{2 k-2}\right)+h\left(v_{k}\right) \\
= & 1+1+1=3 \neq 1 .
\end{aligned}
$$

Therefore $h$ is not a total unidominating function.

Define another function $h_{1}: V \rightarrow\{0,1\}$ by

$h_{1}(u)=f(u) \forall u \in V, u \neq w_{2 k-1}, w_{2 k}, k \in\{1,2, \ldots, n\}$,

and $\mathrm{h}_{1}\left(\mathrm{w}_{2 \mathrm{k}-1}\right)=0, \mathrm{~h}_{1}\left(\mathrm{w}_{2 \mathrm{k}}\right)=0$.

Obviously $\mathrm{h}_{1}<f$.

Now $\begin{aligned} \sum_{u \in N\left(v_{k}\right)} h_{1}(u) & =h_{1}\left(u_{k}\right)+h_{1}\left(w_{2 k-1}\right)+h_{1}\left(w_{2 k}\right) \\ & =0+0+0=0 \neq 1 .\end{aligned}$

Therefore $h_{1}$ is not a total unidominating function.
Since $g, h, h_{1}$ are defined arbitrarily, and there is no other possibility of defining a total unidominating function $<f$ it follows that $f$ is a minimal total unidominating function.

$$
\text { Now } \begin{gathered}
f(V)=\sum_{i=1}^{n}\left[f\left(u_{i}\right)+f\left(v_{i}\right)+f\left(w_{2 i-1}\right)+f\left(w_{2 i}\right)\right] \\
=0+n+n+n=3 n .
\end{gathered}
$$

Therefore $\Gamma_{t u}(3-$ regularized wheel $) \geq 3 n----(1)$

Let $f$ be a minimal total unidominating function.

Then $f\left(u_{i}\right)=f\left(v_{i}\right)=f\left(w_{2 i-1}\right)=f\left(w_{2 i}\right)=1$ is possible only for at most two consecutive $i$ 's.

Let $k_{1}$ be the number of $u_{i} s$ such that

$f\left(u_{i}\right)=0$ and any one of $f\left(u_{i-1}\right), f\left(u_{i+1}\right)$ is 1 and $k_{2}$ be the number of $u_{i} s$ such that $f\left(u_{i}\right)=0$ and

$$
\begin{aligned}
& f\left(u_{i-1}\right)=f\left(u_{i+1}\right)=0 \\
& \text { Then } \sum_{\mathrm{n}-\left(k_{1}+k_{2}\right)}\left[f\left(v_{i}\right)+f\left(w_{2 i-1}\right)+f\left(w_{2 i}\right)\right] \\
& \leq 3\left(\frac{2\left(\mathrm{n}-\left(k_{1}+k_{2}\right)\right)}{3}\right)
\end{aligned}
$$

and $f(V) \leq n-\left(k_{1}+k_{2}\right)+2\left(\mathrm{n}-\left(k_{1}+k_{2}\right)\right)+k_{1}+3 k_{2}$

$$
=3 n-2 k_{1} \leq 3 n \text {. }
$$

Since $f$ is arbitrary, it follows that $\Gamma_{t u}(3-$ regularized wheel $) \leq 3 n---(2)$

Thus from the inequalities (1) and (2) we have

$$
\Gamma_{t u}(3-\text { regularized } \text { wheel })=3 n \text {. }
$$

Theorem 4.2: The number of minimal total unidominating functions of a 3-regularized wheel with maximum weight is

$$
\begin{array}{r}
\text { when } n \equiv 0(\bmod 3), \\
\text { when } n \equiv 1,2(\bmod 3) .
\end{array}
$$

Proof: Consider the minimal total unidominating function $f$ with maximum weight $3 n$ given by

$$
f(u)=\left\{\begin{array}{rr}
1 & \text { for } u=v_{i}, w_{2 i-1}, w_{2 i}, i=1,2, \ldots, n, \\
0 & \text { for } u=u_{i} i=1,2, \ldots, n .
\end{array}\right.
$$

By rotating the functional values of $f$ taking $u_{i}, v_{i}, w_{2 i-1}, w_{2 i}$ as one group we get the same function. Therefore there is one and only one minimal total unidominating function with maximum weight $3 n$.

Now we investigate for some other minimal total unidominating functions with maximum weight $3 n$.

Let $f$ be a minimal total unidominating function. Then we have proved in Theorem 4.1 that $f(V) \leq 3 n-2 k_{1}$.

If $k_{1}>0$ then $f(V) \leq 3 n-2 k_{1}<3 n$. Therefore $f$ is not a function with maximum weight.

If $k_{1}=0$ then $k_{2}=n$ or $k_{2}=0$.

Suppose $k_{2}=n$. Then $f(V)=3 n$ and this function coincides with the above said function.

Suppose $k_{2}=0$. Then $f\left(u_{i}\right)=1 \forall i=1,2, \ldots, n$.

Based on the condition 
$f\left(u_{i}\right)=f\left(v_{i}\right)=f\left(w_{2 i-1}\right)=f\left(w_{2 i}\right)=1$ is possible only for at most two consecutive $i$ 's we get another minimal total unidominating function with weight $3 n$ when $n \equiv 0(\bmod 3)$.

This function is defined by

$\mathrm{f}(\mathrm{u})$

$=\left\{\begin{array}{c}1 \\ 0\end{array}\right.$ for $u=u_{i}, i=1,2, \ldots, n$ and $u=v_{i}, w_{2 i-1}, w_{2 i}, i \equiv 1,2(\bmod 3)$,

By rotating the functional values of $f$ taking $u_{i}, v_{i}, w_{2 i-1}, w_{2 i}$ as one group we get two other minimal total unidominating functions with maximum weight. Therefore there are $1+3=4$ minimal total unidominating functions with maximum weight $3 n$ when $n \equiv 0(\bmod 3)$.

When $n \equiv 1,2(\bmod 3)$ the total unidominating functions obtained has weight $<3 n$ and hence there is no other function with maximum weight.

Hence the number of minimal total unidominating functions with maximum weight $3 \mathrm{n}$ is 4 when $n \equiv 0(\bmod 3)$ and 1 when $n \equiv 1,2(\bmod 3)$.

\section{ILLUSTRATIONS}

In this section the examples for upper unidominating functions and upper total unidominating functions are given.

Example 5.1: Let $n=8$.

Clearly $8 \equiv 2(\bmod 3)$.

The functional values of a minimal unidominating function defined in Case 1 of Theorem 3.1 are denoted at the corresponding vertices in Fig. 1.

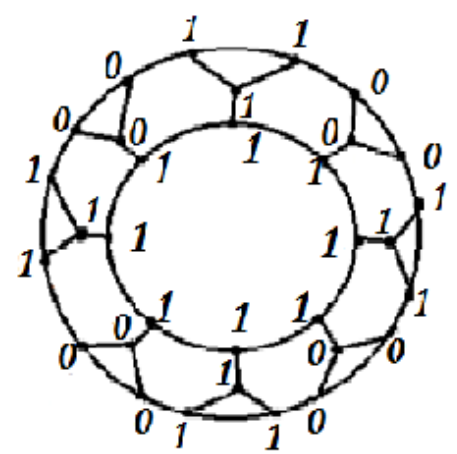

Fig. 1 3-regularized wheel formed from $\mathrm{W}_{1,8}$

Upper unidomination number of 3-regularized wheel formed from $W_{1,8}$ is

$\Gamma_{\mathrm{u}}(3-$ regularized wheel $)$ is $\frac{5 \mathrm{n}}{2}=20$.

There are 2 minimal unidominating functions with maximum weight 20 .

Example 5.2: Let $\mathrm{n}=15$.

Clearly $15 \equiv 0(\bmod 3)$.

The functional values of a minimal unidominating function defined in Case 2 of

Theorem 3.1 are denoted at the corresponding vertices in Fig. 2

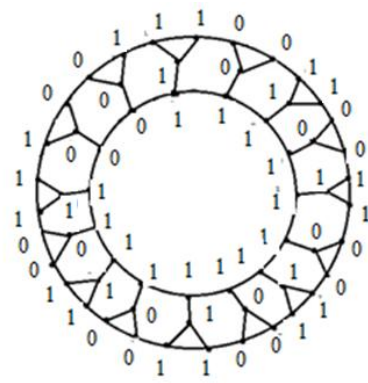

Fig. 2 3-regularized wheel formed from $W_{1,15}$

Upper unidomination number of 3-regularized wheel formed fromW $_{1,15}$ is

$\Gamma_{\mathrm{u}}(3-$ regularized wheel $)=\left\lfloor\frac{5 \mathrm{n}}{2}\right\rfloor-1=37-1=36$.

There are 15 minimal unidominating functions with maximum weight 36 .

Example 5.3: Let $n=8$.

The functional values of the minimal total unidominating function defined in Theorem 4.1 of a 3-regularized wheel formed from $\mathrm{W}_{1,8}$ are given at the corresponding vertices in Fig. 3

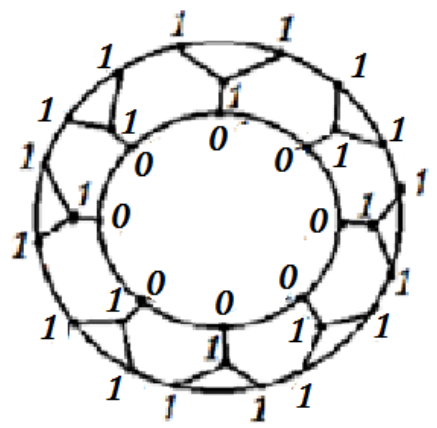

Fig. 3 3-regularized wheel formed from $W_{1,8}$

Upper total unidomination number of this 3-regularized wheel is 24 .

There is only one total unidominating function with maximum weight.

\section{CONCLUSION}

3-regularized wheel formed from $\mathrm{W}_{1, \mathrm{n}}$ is new concept introduced by the authors and in this paper the upper unidomination number of a 3-regularized wheel and number of minimal unidominating functions with maximum weight is calculated based on $n$ is even or odd. Further, the number of minimal total unidominating functions with maximum weight is based on whether $n \equiv 0(\bmod 3)$ or otherwise. This gives scope for further study on this new graph and various domination parameters can be enumerated.

\section{REFERENCES}

[1] Haynes, T.W. Hedetniemi, S.T. Slater, P.J. Fundamentals of domination in graphs, Marcel Dekker, Inc., New York (1998).

[2] Haynes, T. W., Hedetniemi, S.T., Slater, P.J., Domination in graphs: Advanced Topics, Marcel Dekker, Inc., New York (1998). 
[3] Hedetniemi, S.M., Hedetniemi, S.T., Wimer, T.V. Linear time resource allocation algorithm for trees. Technical report URI - 014, department of Mathematics, Clemson University (1987)

[4] Zelinka, B. Some remarks on domination in cubic graphs, Disc.Math. 158 (1996), pp-249-255.

[5] V. Anantha Lakshmi, B. Maheswari - Unidominating functions of a path: International Journal of Computer Engineering \& Technology (IJCET), Volume 6, Issue 8, Aug 2015, pp. 11-19.

[6] Anantha Lakshmi, V and Maheswari, B. - Total Unidominating Functions of a Path:International Journal of Computer Applications ( IJCA), vol 126-No.13, (2015), pp-43-48.

[7] V. Anantha Lakshmi, B. Maheswari - Unidominating functions of a complete k-partite graph: International
Journal of Scientific \& Engineering Research (IJSER), Volume 6, Issue 11, November-2015, Pp 1256-1261.

[8] V. Anantha Lakshmi, B. Maheswari - Total Unidominating functions of a complete k-partite graph: Open Journal of Applied \& Theoretical Mathematics, Vol.2, No.4, December, 2016 pp.795-805

[9] V. Anantha Lakshmi, B. Maheswari - Unidominating functions and Unidomination number of a 3-regularized wheel: International Journal of Research in Science and Engineering (IJRSE) Vol.2, issue:3 March-April, 2017, pp-207-214.

[10] V. Anantha Lakshmi, B. Maheswari - Total Unidominating Functions and Total Unidomination Number of a 3-Regularized Wheel: International Journal of Current Engineering and Scientific Research (IJCESR) Volume 5, Issue 4, April, 2018, pp-82-85 